\title{
Tumor Infiltrating Neutrophils Are Frequently Found in Adenocarcinomas of the Biliary Tract and Their Precursor Lesions with Possible Impact on Prognosis
}

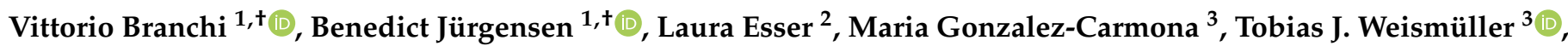

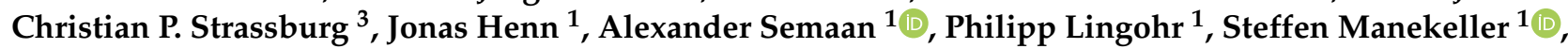 \\ Glen Kristiansen ${ }^{2}$, Jörg C. Kalff ${ }^{1}$, Marieta I. Toma ${ }^{2}$ and Hanno Matthaei ${ }^{1, *}$ \\ 1 Department of General, Visceral, Thoracic and Vascular Surgery, University Hospital Bonn, \\ 53127 Bonn, Germany; vittorio.branchi@ukbonn.de (V.B.); benedict.juergensen@ukbonn.de (B.J.); \\ jonas.henn@ukbonn.de (J.H.); alexander.semaan@ukbonn.de (A.S.); philipp.lingohr@ukbonn.de (P.L.); \\ steffen.manekeller@ukbonn.de (S.M.); joerg.kalff@ukbonn.de (J.C.K.) \\ 2 Institute of Pathology, University Hospital Bonn, 53127 Bonn, Germany; laura.esser@ukbonn.de (L.E.); \\ glen.kristiansen@ukbonn.de (G.K.); marieta.toma@ukbonn.de (M.I.T.) \\ 3 Department of Internal Medicine I, University Hospital Bonn, 53127 Bonn, Germany; \\ maria.gonzalez-carmona@ukbonn.de (M.G.-C.); tobias.weismueller@ukbonn.de (T.J.W.); \\ christian.strassburg@ukbonn.de (C.P.S.) \\ check for \\ updates \\ * Correspondence: hanno.matthaei@ukbonn.de \\ + These authors contributed equally to this work.
}

Citation: Branchi, V.; Jürgensen, B.; Esser, L.; Gonzalez-Carmona, M.; Weismüller, T.J.; Strassburg, C.P.; Henn, J.; Semaan, A.; Lingohr, P.; Manekeller, S.; et al. Tumor Infiltrating Neutrophils Are Frequently Found in

Adenocarcinomas of the Biliary Tract and Their Precursor Lesions with Possible Impact on Prognosis. J. Pers. Med. 2021, 11, 233. https://doi.org/ $10.3390 /$ jpm11030233

Academic Editor: Ari VanderWalde

Received: 23 January 2021

Accepted: 15 March 2021

Published: 23 March 2021

Publisher's Note: MDPI stays neutral with regard to jurisdictional claims in published maps and institutional affiliations.

Copyright: () 2021 by the authors. Licensee MDPI, Basel, Switzerland. This article is an open access article distributed under the terms and conditions of the Creative Commons Attribution (CC BY) license (https:// creativecommons.org/licenses/by/ $4.0 /)$.

\begin{abstract}
Biliary tract cancer (BTC) is characterized by an intense stromal reaction and a complex landscape of infiltrating immune cells. Evidence is emerging that tumor-infiltrating neutrophils (TINs) have an impact on carcinogenesis and tumor progression. TINs have also been associated with outcomes in various solid malignant tumors but their possible clinical role in BTC is largely unknown. Tissue samples from patients with sporadic BTC ("spBTC" cohort, $N=53$ ) and BTC in association with primary sclerosing cholangitis ("PSC-BTC" cohort, $N=7$ ) were collected. Furthermore, tissue samples from 27 patients with PSC who underwent liver transplantation ("PSC-LTX" cohort) were investigated. All specimens were assessed for TIN density in invasive and precancerous lesions (biliary intraepithelial neoplasia, BilIN). Most spBTC showed low TIN density (LD, 61\%). High TIN density (HD) was detected in $16 \%$ of the tumors, whereas $23 \%$ were classified as intermediate density (ID); the majority of both HD and ID groups were in T1-T2 tumors ( $83 \%$ and $100 \%, p=0.012)$. TIN density in BilIN lesions did not significantly differ among the three groups. The HD group had a mean overall survival (OS) of 53.5 months, whereas the mean OS in the LD and ID groups was significantly shorter (LD 29.5 months vs. ID 24.6 months, log-rank $p<0.05$ ). The results of this study underline the possible prognostic relevance of TINs in BTC and stress the complexity of the immune cell landscape in BTC. The prognostic relevance of TINs suggests a key regulator role in inflammation and immune landscape in BTC.
\end{abstract}

Keywords: cholangiocarcinoma; biliary tract cancer; prognosis; neutrophils

\section{Introduction}

Biliary tract cancers (BTC) are a heterogeneous group of malignancies arising from the epithelial cells of the intra- and extrahepatic biliary ductal system and gallbladder. These tumors account for approximately $3 \%$ of all gastrointestinal cancers and represent the second most common primary liver tumor [1,2]. Hence, BTC are rare, while in particular the incidence of intrahepatic cholangiocarcinoma is on the rise in Western countries [3]. BTC is one of the most aggressive cancer entities and radical surgery represents the only curative option [4]. However, only about $30 \%$ of patients with BTC are resectable at the 
time of first presentation [5]. Even after radical surgery, the median overall survival barely reaches three years [6].

Several risk factors for the development of BTC have been identified, such as chronic inflammation through primary sclerosing cholangitis (PSC), viral hepatitis, liver cirrhosis, and liver fluke [7]. In fact, BTC is histologically characterized by an intense stromal reaction while the stroma is densely populated by cancer-associated fibroblasts (CAF) and various infiltrating immune cells such as monocytes and tumor-associated macrophages (TAM). These inflammatory cells likely play a predominant role in tumor development and progression $[8,9]$. Thus, a biomarker function of easily obtainable systemic inflammation markers such as C-reactive protein (CRP), neutrophils to lymphocytes ratio (NLR), and platelets to lymphocytes ratio (PLR) has been intensely investigated in BTC in the recent years with remarkable success [10-14]. Neutrophils are the most abundant white blood cell subtype, thereby representing a crucial component of the innate immune system. While the main function of neutrophils is to fight infections by phagocytosis and elimination of microorganisms, a chronic neutrophilic inflammation has been associated with the early phase of various epithelial cancers. Interestingly, evidence is emerging that tumor-infiltrating neutrophils (TINs) are impacting progression and spread in later phases of the disease. Apparently, malignant tumors have the ability to induce myelopoiesis and to attract neutrophils to the tumor microenvironment. Here, these cells gather a protumorigenic, immunosuppressive phenotype, whereas the exact mechanisms and possible targeted therapies are currently under investigation [15]. Not surprisingly, TINs have been associated with outcome in various solid tumors $[16,17]$. However, their presence and possible prognostic role in BTC is still largely unknown and focus of the present study.

\section{Methods}

\subsection{Patients and Tissues}

For this monocentric retrospective study, data from three different patient cohorts were collected and analyzed.

\subsubsection{Patients with Surgically Resected Sporadic Biliary Tract Cancer (spBTC Cohort)}

Tissue samples from 53 patients who underwent surgical resection for sporadic BTC (spBTC) between 2013 and 2018 at the Department of Surgery, Bonn University Hospital, were collected. Only patients without previous radiochemotherapy were selected. All patients were operated on in the absence of clinical signs of an acute infection. Patient serum was obtained shortly before surgery and analyzed for carcinoembryonic antigen (CEA), carbohydrate antigen 19.9 (CA19.9), and C reactive protein (CRP). Therapy options for every patient were discussed in our weekly interdisciplinary tumor board and all patients included were offered surgical resection. Demographic and clinical data, including age, gender, postoperative complications, hospital stay, and adjuvant chemotherapy were retrieved from the patients' records. Tumor samples from the resected specimens were fixed in formalin and embedded in paraffin (FFPE) according to a standardized protocol. Survival data were available for all patients. The usage of archived diagnostic left-over tissues for tissue microarray (TMA) manufacturing, the analysis for research purposes, and patient data analysis were approved by the ethics committee, Bonn University Hospital (IRB number: 417/17). The study was carried out in compliance with the Helsinki Declaration.

\subsubsection{Patients with Primary Sclerosing Cholangitis and BTC (PSC-BTC Cohort)}

Tissue samples from 7 patients with primary sclerosing cholangitis and BTC (PSC-BTC) were retrieved from archived diagnostic left-over FFPE tissue. All patients were operated on between 2010 and 2017 at the Department of Surgery, Bonn University Hospital. All tumor samples were obtained for diagnostic reasons during radical tumor resection or explorative laparotomy and open biopsy. A survival analysis was not performed in this cohort due to the small number of patients. 


\subsubsection{Patients with PSC Who Underwent Liver Transplantation (PSC-LTX Cohort)}

Tissue samples from 27 patients with PSC who underwent liver transplantation between 2003 and 2017 at the Department of Surgery, Bonn University Hospital, were collected from FFPE left-over tissue. A mean of 12 samples per patient, each from different localizations along the biliary ductal system, were analyzed. A survival analysis was not performed in this cohort due to the small number of patients.

\subsection{Tissue Microarray Construction and Tumor-Infiltrating Neutrophil (TIN) Density Score Analysis in Sporadic BTC}

For spBTC, a tissue microarray (TMA) was constructed according to standardized protocols. Briefly, four to six different $1 \mathrm{~mm}$ cores were taken from every tissue sample. For internal controls, normal kidney and normal liver tissue cores were included in each TMA block. A $2 \mu \mathrm{m}$ section was stained following a standard hematoxylin and eosin staining protocol. Neutrophils were counted in each core by an experienced pathologist, who was blinded to tumor stage and patient characteristics. Apoptotic neutrophils, as well as neutrophils found inside the lumen of blood vessels were excluded. Then, the mean value was calculated for each tumor, as well as the 60 and the 85 percentile which we used as cut-offs. The use of these cut-offs was arbitrary in order to classified samples into "low density" if the TIN density score was lower than the 60th percentile (LD, score 0), "intermediate density", if the TIN density score was between the 60th and 85th percentiles (ID, score 1), or "high density" if the TIN density score was higher than the 85th percentile (HD, score 2).

\subsection{Neutrophils Density Analysis in Biliary Intraepithelial Neoplasia (BilIN)}

For spBTC and PSC-BTC cohorts, diagnostic slides were reviewed for the presence of high-grade biliary intraepithelial neoplasia (BilIN) in the tumor proximity. For the PSC patients with PSC who underwent liver transplantation (PSC-LTX) cohort, all diagnostic slides were reviewed for the presence of high-grade BilIN in bile ducts. In the presence of multiple BilIN lesions, one representative high-grade BilIN was randomly selected. The total number of neutrophils in six $40 \times$ microscopic fields (three intraepithelial/intratumoral fields and three in periepithelial stroma) were assessed. Neutrophils that were identified in blood vessels were not counted. The mean value for each localization was then calculated.

\subsection{BTC and BilIN Classification}

All tumors were reclassified according to the UICC (Union for International Cancer Control) TNM classification system by the International Union Against Cancer, 8th Edition [18]. BilINs were classified according to established histopathological characteristics such as degree of cellular and structural atypia as recommended $[19,20]$.

\subsection{Statistical Analysis}

Statistical analysis was performed in the R environment (RStudio Version 1.3, package survminer version 0.4.8) and SPSS Statistics Version 22 (IBM, Armonk, New York, NY, USA) [21,22]. Continuous variables are shown as mean or median with interquartile range (IQR). Univariate analysis was performed, and Kaplan-Meier (KM) plots were generated for overall survival (OS). KM curves were compared using the log-rank test. Multivariate analyses for OS were performed using the Cox regression method. Variables which were significant in the univariate analysis were included in the Cox regression model. Mean OS was indicated if the median OS was not reached. Comparisons between groups were made with Fisher's exact test or Anova test, as appropriate. Pearson's correlation analysis for TIN density in BillN was performed and displayed as scatter plot. Statistical significance was assumed at a $p$-value $<0.05$. 


\section{Results}

\subsection{Patient Characteristics}

A total of 53 patients (29 females and 24 males, median age 67, range 38-81) were enrolled in the spBTC cohort. A majority of tumors was diagnosed as intrahepatic cholangiocarcinoma (IHC, $N=19,36 \%$ ), followed by distal cholangiocarcinoma (DC, $N=14,26 \%$ ), and perihilar cholangiocarcinoma (PHC, $N=13,24 \%$ ). A gallbladder carcinoma (GBCA) was diagnosed in seven patients (13\%). Median preoperative CA19.9 and CEA levels were $53.6 \mathrm{kU} / \mathrm{L}$ (IQR 26.9-359 kU/L) and $2.2 \mathrm{ng} / \mathrm{mL}$ (IQR 1.2-3.0 ng/mL), respectively. Median preoperative CRP level was $17.2 \mathrm{mg} / \mathrm{L}$ (IQR 7.2-59.5 mg/L). Most primary tumors were classified pT2 $(N=19,36 \%)$ or pT3 $(N=19,36 \%)$, whereas $28 \%$ of the patients were staged pT1 $(N=15,28 \%)$. A positive nodal status was found in $57 \%$ of the patients $(\mathrm{N} 1, N=30)$. Four patients (7\%) with apparently resectable disease had to be classified M1 according to the final pathology report due to peritoneal carcinomatosis. Most tumors were moderately differentiated (G2, $N=31,58 \%$ ). Postoperative adjuvant chemotherapy was administered in a majority of patients $(N=33,62 \%)$. Characteristics of the spBTC cohort are summarized in Table 1.

Table 1. Patient's characteristics according to the extent of tumor-infiltrating neutrophils (TINs) in resected biliary tract cancer (BTC).

\begin{tabular}{|c|c|c|c|c|c|c|}
\hline & & & & TIN Density & & \\
\hline & & $\begin{array}{c}\text { All Patients } \\
N=53\end{array}$ & $\begin{array}{c}\text { Low } \\
N=31\end{array}$ & $\begin{array}{c}\text { Intermediate } \\
\quad N=12\end{array}$ & $\begin{array}{l}\text { High } \\
N=8\end{array}$ & \\
\hline & & $\begin{array}{c}n, \text { mean, median } \\
(\%, S D, \text { or IQR })\end{array}$ & $\begin{array}{c}n, \text { mean, median } \\
(\%, S D \text {, or IQR })\end{array}$ & $\begin{array}{c}n, \text { mean, median } \\
(\%, S D \text {, or IQR })\end{array}$ & $\begin{array}{c}n, \text { mean, median } \\
(\%, S D \text {, or IQR })\end{array}$ & $p$ \\
\hline Sex & $\begin{array}{l}\mathrm{W} \\
\mathrm{M}\end{array}$ & $\begin{array}{l}29(54.7) \\
24(45.3)\end{array}$ & $\begin{array}{l}18(58.1) \\
13(41.9)\end{array}$ & $\begin{array}{l}5(41.7) \\
7(58.3)\end{array}$ & $\begin{array}{l}4(50.0) \\
4(50.0)\end{array}$ & 0.624 \\
\hline & $\leq 67$ & $28(52.8)$ & $16(51.6)$ & $7(58.3)$ & $4(50.0)$ & \\
\hline Age & $>67$ & $25(47.2)$ & $15(48.4)$ & $5(41.7)$ & $4(50.0)$ & 0.925 \\
\hline CA19-9 & & $53.6(26.9-359.0)$ & $53.6(28.3-160.4)$ & $61.8(20.3-360.4)$ & $36.4(29.7-1164.6)$ & 0.325 \\
\hline CEA & & $2.2(1.2-3.0)$ & $2.4(1.4-3.3)$ & $2.2(1.1-4.6)$ & $1.2(0.9-1.3)$ & 0.080 \\
\hline CRP & & $17.2(7.2-59.5)$ & $24.7(9.50-75.50)$ & $11.0(4.9-31.2)$ & $19.4(6.2-26.7)$ & 0.396 \\
\hline \multirow{4}{*}{ Localization } & IHC & $19(35.8)$ & $10(32.3)$ & $5(41.7)$ & $4(50.0)$ & \multirow{4}{*}{0.312} \\
\hline & PHC & $13(24.5)$ & $6(19.4)$ & $2(16.7)$ & $3(37.5)$ & \\
\hline & $\mathrm{DC}$ & $14(26.4)$ & $8(25.8)$ & 5 (41.7) & $1(12.5)$ & \\
\hline & GBC & $7(13.2)$ & $7(22.6)$ & $0(0.0)$ & $0(0.0)$ & \\
\hline & $\mathrm{T} 1$ & $15(28.3)$ & $6(19.4)$ & 4 (33.3) & $4(50.0)$ & \multirow{4}{*}{0.012} \\
\hline \multirow{3}{*}{$\mathrm{T}$} & T2 & 19 (35.8) & $8(25.8)$ & $6(50.0)$ & $4(50.0)$ & \\
\hline & T3 & $19(35.8)$ & $17(54.8)$ & $2(16.7)$ & $0(0.0)$ & \\
\hline & $\mathrm{T} 4$ & $0(0)$ & $0(0.0)$ & $0(0.0)$ & $0(0.0)$ & \\
\hline \multirow{2}{*}{$\mathrm{N}$} & No & $23(43.4)$ & 9 (29.3) & $9(75.0)$ & $4(50)$ & \multirow{2}{*}{0.025} \\
\hline & N1 & $30(56.6)$ & $22(71.0)$ & $3(25.0)$ & $4(50)$ & \\
\hline \multirow{2}{*}{$\mathrm{M}$} & M0 & $49(92.5)$ & $27(87.1)$ & $12(100.0)$ & $8(100.0)$ & \multirow{2}{*}{0.462} \\
\hline & M1 & $4(7.5)$ & $4(12.9)^{\prime}$ & $0(0.0)$ & $0(0.0)$ & \\
\hline \multirow[b]{2}{*}{$\mathrm{L}$} & L0 & 39 (73.6) & $22(71.0)$ & $9(75.0)$ & $7(87.5)$ & \multirow{2}{*}{0.743} \\
\hline & L1 & $14(26.4)$ & $9(29.0)$ & $3(25.0)$ & 1 (12.5) & \\
\hline \multirow{2}{*}{ V } & V0 & $44(83.0)$ & $24(77.4)$ & $11(91.7)$ & $7(87.5)$ & \multirow{2}{*}{0.671} \\
\hline & V1 & $9(17.0)$ & $7(22.6)$ & $1(8.3)$ & $1(12.5)$ & \\
\hline \multirow{3}{*}{ Pn } & Pn0 & $24(45.3)$ & $13(41.9)$ & $6(50.0)$ & $3(37.5)$ & \multirow{3}{*}{0.851} \\
\hline & Pn1 & $29(54.7)$ & 18 (58.9) & $6(50.0)$ & $5(62.5)$ & \\
\hline & G1 & $3(5.7)$ & $2(6.5)$ & $1(8.3)$ & $0(0.0)$ & \\
\hline \multirow[t]{2}{*}{ G } & G2 & 31 (58.5) & $18(58.1)$ & $8(66.7)$ & $3(37.5)$ & \multirow{2}{*}{0.517} \\
\hline & G3 & $19(35.8)$ & $11(35.5)$ & $3(25.0)$ & $5(62.5)$ & \\
\hline \multirow{3}{*}{$\mathrm{R}$} & R0 & $42(79.2)$ & $25(80.6)$ & $11(91.7)$ & $5(62.5)$ & \multirow{3}{*}{0.285} \\
\hline & $\mathrm{R}+$ & $11(20.8)$ & $6(19.4)$ & $1(8.3)$ & $3(37.5)$ & \\
\hline & Stage I & $9(17.0)$ & $1(3.2)$ & $4(33.3)$ & $3(37.5)$ & \\
\hline \multirow{3}{*}{ Stage } & Stage II & $19(35.8)$ & $12(38.7)$ & $6(50.0)$ & 1 (12.5) & \multirow{3}{*}{0.010} \\
\hline & Stage III & $20(37.7)$ & $14(45.2)$ & $1(8.3)$ & $4(50.0)$ & \\
\hline & Stage IV & $5(9.4)$ & $4(12.9)$ & $1(8.3)$ & $0(0.0)$ & \\
\hline Postoperative & Yes & $28(52.8)$ & $16(51.6)$ & $6(50.0)$ & $5(62.5)$ & \multirow{2}{*}{0.837} \\
\hline Complications & No & $25(47.2)$ & $15(48.4)$ & $6(50.0)$ & $3(37.5)$ & \\
\hline \multirow{2}{*}{ Chemotherapy } & Yes & $33(62.3)$ & $22(71.0)$ & $6(50.0)$ & $4(50.0)$ & \multirow{2}{*}{0.356} \\
\hline & No & $20(37.7)$ & $9(29.0)$ & $6(50.0)$ & $4(50.0)$ & \\
\hline
\end{tabular}




\subsection{Correlation of TIN Density with Clinicopathologic Parameters}

Among the tumor samples, $16 \%$ were classified as tumors with a high TIN density $(N=8)$, while $23 \%$ were classified as intermediate $(N=12)$, and $61 \%$ were classified as low TIN-density tumors $(N=31)$. Two samples had to be excluded from the analysis because of tissue fragmentation during TMA preparation. Representative images of tumor sections with different TIN densities are displayed in Figure 1.

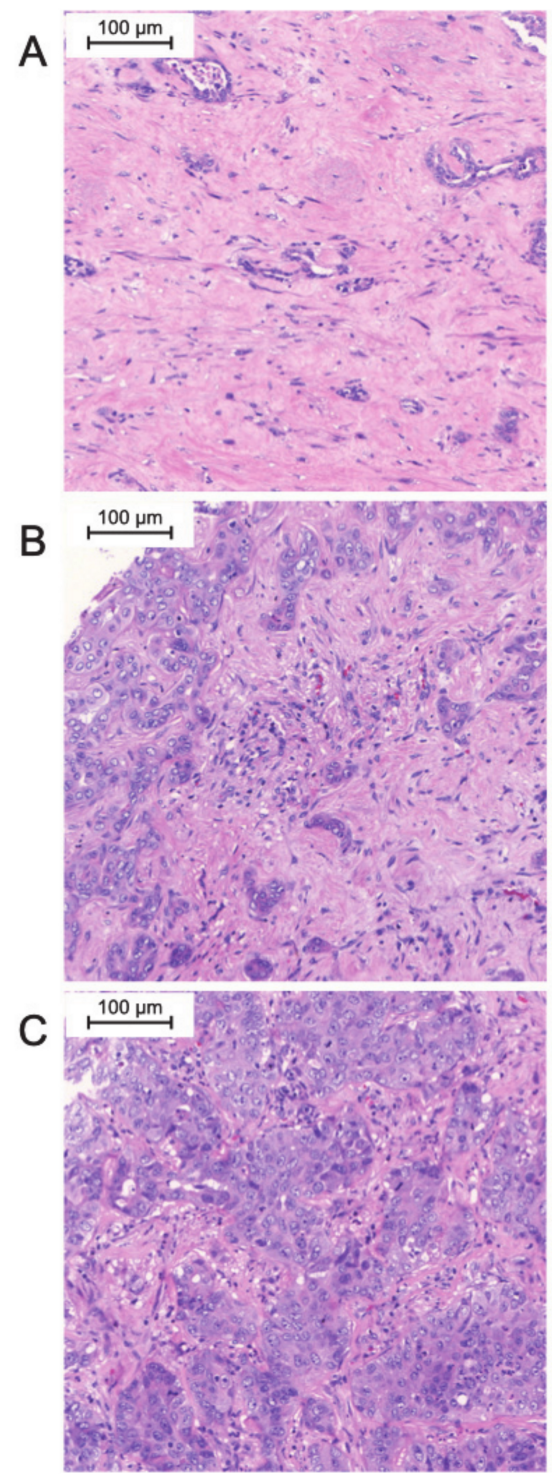

Figure 1. Examples of high-resolution microscopy images of biliary tract cancer with low (A), intermediate (B), and high (C) TIN density (hematoxylin and eosin staining).

The demographics of the three groups were similar regarding age (mean age 67 in the LD group vs. 66 in the ID group vs. 67 in the HD group) and gender (58\% females and $42 \%$ males in the LD group vs. $42 \%$ females and $58 \%$ males in the ID group and $50 \%$ females vs. $50 \%$ males in the HD group). Tumor stage distribution differed significantly in the three groups, since in the LD group the most represented T stage was T3 $(N=17$, $55 \%)$. In the ID group, the majority of the patients had T1-T2 tumors $(N=10,83 \%)$ and $17 \%(N=2)$, also in the HD group, all had a comparatively smaller T1-T2 primary tumor $(p=0.012)$. The rate of $\mathrm{N}+$ tumors was also significantly different in the three groups. In the LD group, $71 \%(N=22)$ had a positive nodal status as compared with $25 \%(N=3)$ in the ID group and $50 \%(N=4)$ in the HD group $(p=0.025)$. No difference was observed 
regarding preoperative CA19.9, CEA, CRP, tumor location, $\mathrm{M}$ stage, grading, as well as lymphovascular and perineural invasion. Most of the tumors in the LD were IHC ( $N=10$, $32 \%)$ followed by DC $(N=8,26 \%)$, GBC $(N=7,23 \%)$, and PHC $(N=6,19 \%)$. In the ID group, IHC and DC were the most represented tumor localizations $(N=5,42 \%)$, followed by PHC $(N=2,17 \%)$. In the HD group, IHC were the most frequent tumors $(N=4,50 \%)$, followed by PHC $(N=3,37 \%)$ and DC $(N=1,12 \%)$ The LD group was the only group with samples from GBCA patients. The abovementioned varying TIN densities according to $\mathrm{T}$ stage and positive nodal status were mirrored in the respective UICC stages. In fact, in the LD group, most patients had advanced UICC stages III or IV, in the ID group the majority of the patients had earlier UICC stages I or II, and in the HD group $50 \%$ had a UICC stage I or II $(p=0.010)$. Clinicopathological characteristics of the three groups are summarized in Table 1.

\subsection{Correlation of TIN Density with Outcome}

Median follow-up time was 19.4 months (range 0.2-60 months). The HD group had a mean OS of 53.5 months (standard error (SE) 6.0, 95\% confidence interval (CI) 41.7-65.4), whereas the mean OS in the LD and ID groups was significantly shorter (LD group, 29.5 months, SE 3.9, 95\% CI 21.7-37.3, log-rank $p<0.05$ and ID group, 24.6 months, SE $6.0,95 \%$ CI 11.8-37.4, log-rank $p<0.05)$. The Kaplan-Meier plot in Figure 2 displays OS probabilities stratified for neutrophil density.

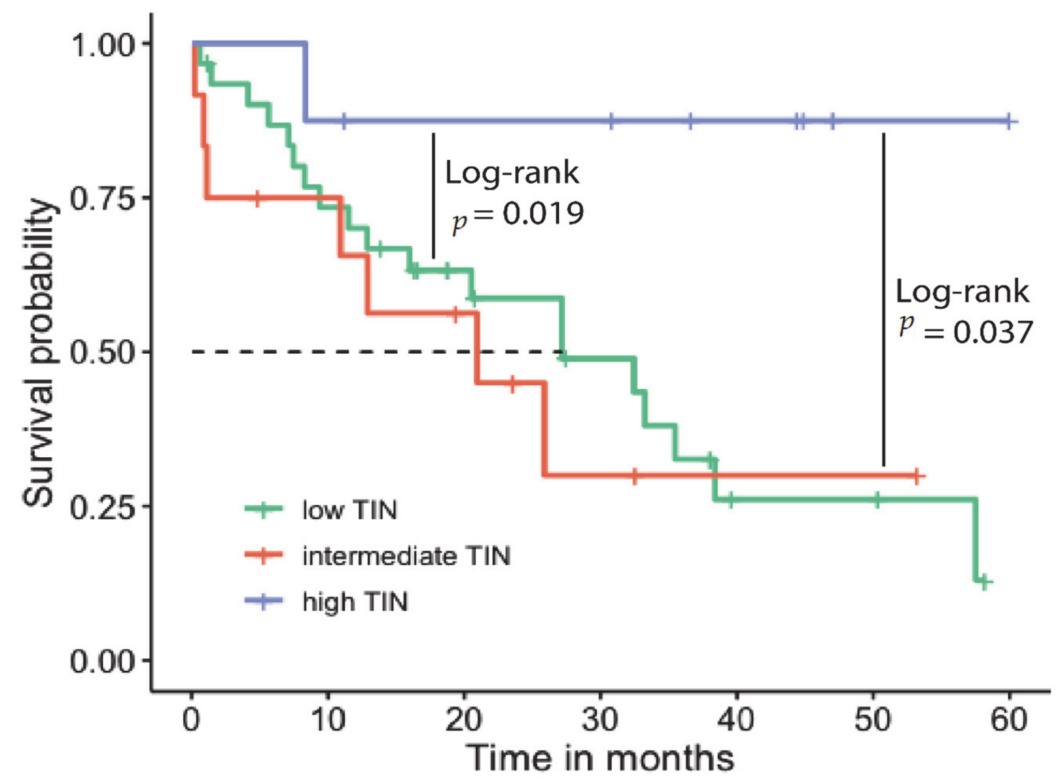

Figure 2. Kaplan-Meier (KM) plot showing survival probability stratified for tumor infiltrating neutrophiles (TIN) density. From the log-rank, only significant $p$-values are displayed.

In the univariate survival analysis, a correlation with poor prognosis in HD vs. ID (hazard ratio (HR) 10.10, 95\% confidence interval (CI) 1.22-83.50, $p=0.032$ ) was found. When comparing HD vs. LD, a similar correlation with prognosis was found (HR 7.66, $95 \%$ CI 1.02-57.53, $p=0.048)$. In addition, univariate analysis showed that a UICC stage III was significantly associated with poorer prognosis as compared with UICC stage I (HR $4.48,95 \%$ CI $1.01-19.92, p=0.049)$. The results of the univariate analyses are summarized in Table 2. The results of the multivariate analysis are summarized in Supplementary Table S1. 
Table 2. Results of the univariate analysis.

\begin{tabular}{cccc}
\hline Subgroup & HR & CI 95\% & $p$ \\
\hline Age $>$ 67 vs. $\leq 67$ & 1.17 & $0.55-2.47$ & 0.678 \\
Female vs. Male & 1.02 & $0.48-2.14$ & 0.963 \\
CEA high vs. low & 1.75 & $0.73-4.20$ & 0.207 \\
CA19.9 high vs. low & 1.78 & $0.82-3.84$ & 0.145 \\
TIN intermediate vs. high & 10.10 & $1.22-83.50$ & 0.032 \\
TIN low vs. high & 7.66 & $1.02-57.53$ & 0.048 \\
Complications Yes vs. No & 1.30 & $0.61-2.78$ & 0.498 \\
G2 vs. G1 & 1.63 & $0.22-12.34$ & 0.636 \\
G3 vs. G1 & 1.72 & $0.22-13.66$ & 0.607 \\
T2 vs. T1 & 0.83 & $0.31-2.23$ & 0.706 \\
T3 vs. T1 & 1.26 & $0.51-3.11$ & 0.612 \\
N1 vs. N0 & 1.69 & $0.79-3.62$ & 0.179 \\
M1 vs. M0 & 1.07 & $0.25-4.53$ & 0.927 \\
Stage 2 vs. Stage 1 & 3.23 & $0.71-14.66$ & 0.128 \\
Stage 3 vs. Stage 1 & 4.48 & $1.01-19.92$ & 0.049 \\
Stage4 vs. Stage 1 & 3.15 & $0.44-22.51$ & 0.254 \\
R1 vs. R0 & 1.30 & $0.52-3.25$ & 0.579 \\
V1 vs. V0 & 1.74 & $0.70-4.34$ & 0.234 \\
L1 vs. L0 & 0.82 & $0.33-2.05$ & 0.676 \\
Pn1 vs. Pn0 & 1.39 & $0.65-2.95$ & 0.394 \\
& 0.62 & $0.29-1.31$ & 0.208 \\
\hline
\end{tabular}

\subsection{Neutrophil Infiltration in BTC-Associated BilIN}

In the spBTC cohort, $23 \%$ of the patients $(N=12)$ had at least one high-grade BilIN lesion in the analyzed slides displaying their primary adenocarcinoma. In the PSC-BTC cohort, $57 \%$ of the patients $(N=4)$ had at least one high-grade BilIN lesion near the tumor. In the PSC-LTX cohort, at least one BilIN lesion was found in $25 \%$ of the explanted liver samples $(N=7)$. The mean TINs in the BilIN lesions from the cohort of patients with PSC who underwent LTX did not significantly differ from the mean TINs of BillN lesions adjacent to sporadic BTC and PSC-associated BTC (7.0, standard deviation (SD) 8.1 vs. 4.9, SD 5.7 vs. 4.0, SD 2.5, $p>0.05$ ) (Figure 3).

A positive correlation between intraepithelial TIN and stromal TIN was observed (Pearson's $R=0.51, p=0.025$ ). The number of TINs in spBTC and PSC-BTC lesions did not correlate with the number of neutrophils infiltrating adjacent BilIN lesions (Pearson's $\mathrm{R}=0.16, p=0.63$ ). Patient characteristics of the PSC-LTX and PSC-BTC cohorts are summarized in the Supplementary Table S2. 
A BillN- and spBTC-associated TIN

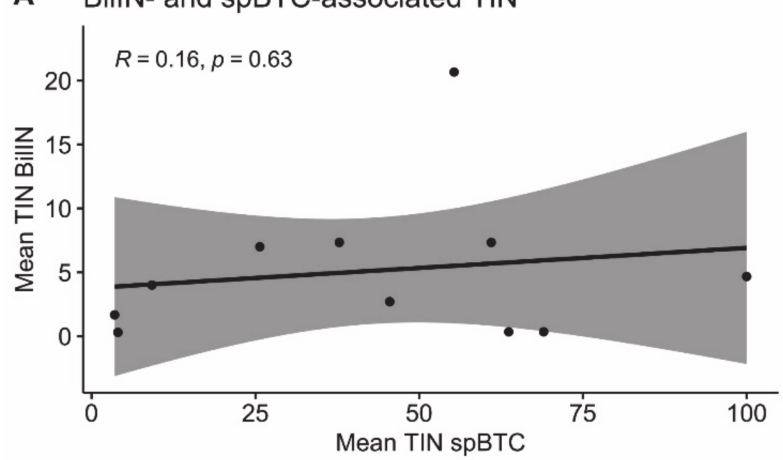

C BillN-associated TIN

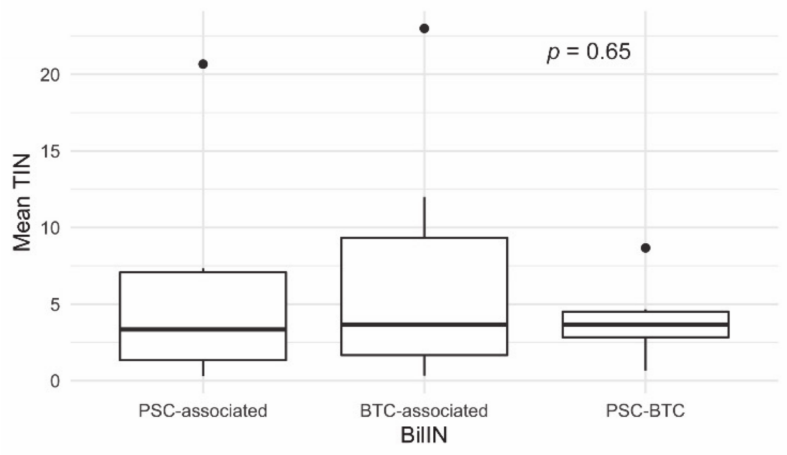

B Intraepithlial and peripheral BillN-associated TIN

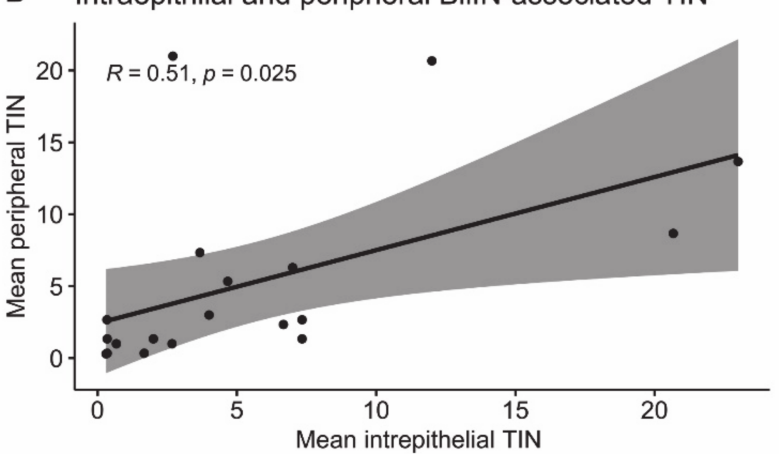

D

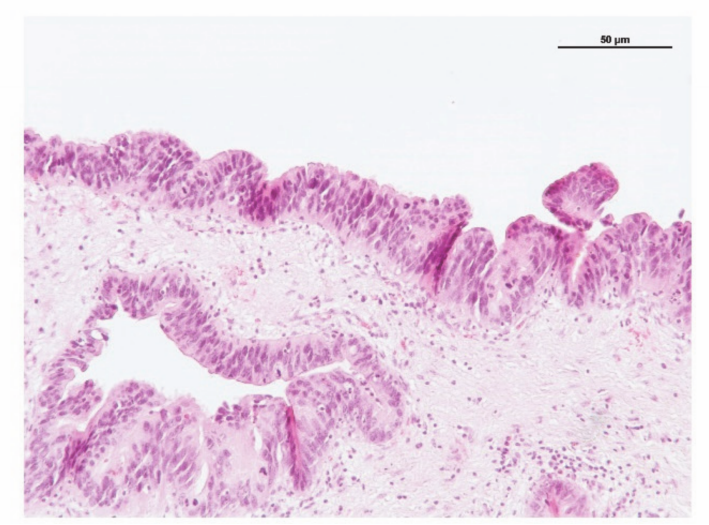

Figure 3. Scatter plot showing the relationship between tumor-infiltrating neutrophils (TINs) in spBTC and BTC-associated biliary intraepithelial neoplasia (BilIN) (A) and between intraepithelial and peripheral (stromal) TINs in BilIN lesions (B). Best-fitting lines and $95 \%$ confidence intervals as well as $\mathrm{R}$ coefficients and $p$ values are displayed. Boxplots displaying mean infiltrating neutrophils (TINs) in BilIN from patients with primary sclerosing cholangitis (PSC), in sporadic BTC-associated BilIN and in PSC-related, BTC-associated BilIN (PSC-BTC). The lower and upper hinges correspond to the 25th and 75th percentiles. The upper/lower whiskers represent the largest/smallest observation less/greater than or equal to upper/lower hinge $+/-1.5$ times the interquartile range (C). Exemplary section of a high-grade BilIN lesion from a patient with BTC. (hematoxylin/eosin staining) (D).

\section{Discussion}

Neutrophils are a crucial component of the human innate immunity and the first circulating cellular responders in the case of acute tissue damage and infections. In cancer, neutrophils play a pivotal role in the tumor microenvironment and can acquire an antitumor (N1) or a protumor (N2) phenotype [23].

Several soluble factors produced by tumor cells promote TIN recruitment. Among others, CXCL1, CXCL2, CXCL5, CXCL6, IL17, IL 8, and CCL3 have been found to act as potent TIN chemoattractants [23]. TIN recruitment is followed by a polarization in either $\mathrm{N} 1$ or N2 phenotype due to a complex cytokine stimulation promoted directly from tumor cells and other tumor microenvironment cells [24]. Transforming growth factor- $\beta$ (TGF- $\beta$ ) and interferon- $\beta$ (IFN- $\beta$ ) have been found to be one of the most important promoters of neutrophil polarization in mice $[25,26]$. Therefore, some studies have addressed the possible use of TGF- $\beta$ inhibition as a therapeutic approach in cancer [26]. However, the N1/2 polarization as well as the role of TINs in human cancers are still controversial [27].

Inflammation and inflammatory mediators are the hallmark of several risk factors associated with BTC [7]. In PSC, chronic inflammation of the bile ducts has been linked to the increased risk of BTC in this population [28]. Choledocholithiasis and cholecystolithiasis are characterized by cholestasis and chronic inflammation and are also considered risk factors for BTC [29]. Liver fluke infections, viral infections, and cirrhosis are similarly linked to cholangiocarcinogenesis [28]. 
Neutrophil infiltration has been evidenced and comprehensively investigated in various solid tumors revealing heterogeneous results. As such, high neutrophil infiltration proved to be related to chemosensitivity and longer recurrence-free survival in high-grade ovarian cancer [30]. Furthermore, high levels of intratumoral granulocytes negatively correlates with cancer-specific survival in patients with clear cell renal cell carcinoma [31]. In hepatocellular carcinoma, neutrophil infiltration has been shown to be a negative prognosticator after curative resection, correlating with angiogenesis progression and tumor recurrence in this entity [32-34]. Ino et al. described similar results in patients with resected adenocarcinomas of the pancreas [35]. The role of TINs has also been investigated in BTC. Gu et al. found that presence of TINs was significantly associated with adverse OS in a cohort of intrahepatic cholangiocarcinomas [36]. Another study found that a high density of CD15 (a carbohydrate epitope expressed on neutrophils) positive neutrophils correlated with shorter OS in cholangiocarcinoma [37]. In our cohort, we observed that patients with tumors with higher neutrophil infiltration had a better prognosis as compared with those with tumors of low or intermediate neutrophil infiltration. Our results regarding OS in resected BTC revealed intriguing aspects. On the one hand, tumors with the highest infiltration showed a longer OS as compared with the group with intermediate infiltration. On the other hand, the group with intermediate TIN infiltration had a tendency of worse OS as compared with the group with low TIN infiltration. However, in the group with high TIN infiltration, there were only T1 and T2 tumors. Moreover, the group with low TINs had a significantly higher $\mathrm{N}+$ tumors as compared with the other groups. This could represent an important confounding factor, and therefore the results of the survival analysis should be interpreted with caution. A multivariate survival analysis of a larger cohort with matched groups could address this topic, provide more robust results, and reduce the bias. The different prognosis among the groups could also be explained based on the complexity of the tumor immune microenvironment in BTC, which includes tumor-associated fibroblasts, tumor-associated macrophages (TAM), dendritic cells, natural killer cells, and myeloidderived suppressor cells. All these cells contribute to both tumor immunosurveillance and immunosuppressive functions. The tumors with high TINs in our cohort may represent a subgroup with a high immunological antitumoral activity. In fact, previous studies have reported that tumor-associated neutrophils could have a dual role. On the one hand, in early tumors, neutrophils could play a role in stimulating T-cell response [38]. On the other hand, neutrophils have an immunosuppressive activity in advanced tumors [39]. In our cohort, the tumors with high TINs were T1 and T2 tumors, whereas in the group with intermediate TIN, T1 to T3 stage tumors were represented. However, this could confirm previous observations about the dual role of TINs in cancer. Interestingly, the number of infiltrating neutrophils in BillNs did not significantly differ from those adjacent to sporadic BTC and those found in patients with PSC with or without BTC. Therefore, it is reasonable to think that neutrophils play a role in the development of a subset of BTC from BilIN towards invasive carcinoma, thus, underlying their tumor-promoting activity in early stage, possibly independent of predisposition.

Our study has some limitations. Firstly, our monocentric cohort was too small to build a proper validation of our preliminary findings which we anticipate in the future. In particular, due to the small BTC cohort and the small number of samples per group, a definitive conclusion about the prognostic role of TINs cannot be reached. A multicentric research effort could potentially address this limitation. Moreover, a multivariate survival analysis of matched groups could help by reducing the bias deriving from unbalanced groups. The retrospective design of this study carries intrinsic limitations, which should be taken into account when interpreting the results. In addition, our study did not focus on further TIN characterization. It is already known that infiltrating neutrophils have different phenotypes and capabilities, which lead to heterogeneous inflammatory responses [40]. This topic has not yet been deeply investigated in BTC and represents an intriguing research field with promising translational implications. Nonetheless, the phenotypical characterization of TINs was not the aim of the present study which mainly focused 
on prognostic implication. Furthermore, we enrolled patients with BTC irrespective of anatomical localization. This may be relevant when assessing prognostic factors for this tumor. However, some authors have described similar outcome for patients with BTC from different localizations but with comparable pathological characteristics [41]. BTCs are believed to be a stem cell-based disease and there are probably several different BTC stem cell populations involved in carcinogenesis [42,43]. In addition, there is growing evidence of a heterogeneous genetic landscape in BTC [44]. Due to the rareness and similar clinical behavior, we should find a prognostic factor that could be useful to the entire population of BTC patients, despite the proven heterogeneity of BTC.

\section{Conclusions}

In summary, the results of this study underline the frequency and possible prognostic relevance of TINs in BTC. Our clinically apparent, though somewhat ambivalent, results stress the complexity of the immune cell landscape in this fatal cancer entity that deserves further scientific dedication. The role of TINs as a prognostic factor in BTC remains unclear. The prognostic relevance of TINs should be further investigated in order to determine if neutrophils may act as a key regulator of inflammation and immune status in BTC. Further studies addressing the role of TINs would hopefully provide new insights to elucidate the associated tumor microenvironment for clinical implications in BTC.

Supplementary Materials: The following are available online at https: / www.mdpi.com/2075-4 426/11/3/233/s1, Table S1: Patient characteristics of the PSC-LTX and PSC-BTC cohort, Table S2: Multivariate analysis.

Author Contributions: Conceptualization, V.B., B.J., M.I.T., and H.M.; Data curation, V.B., B.J., J.H., S.M., M.I.T., and H.M.; Formal analysis, V.B., B.J., L.E., and M.I.T.; Methodology, M.I.T. and H.M.; Project administration, H.M.; Supervision, J.C.K., M.I.T., and H.M.; Writing—original draft, V.B., B.J., L.E., and H.M.; Writing—review and editing, M.G.-C., T.J.W., C.P.S., J.H., A.S., P.L., S.M., G.K., and J.C.K. All authors have read and agreed to the published version of the manuscript.

Funding: V.B. received a post-doctoral fellowship grant from the Else-Kröner Fresenius Stiftung (grant number 2014_Kolleg.05).

Institutional Review Board Statement: All the procedures in this study involving human participants were performed in accordance with the ethical standards of the institutional and national research committee and with the 1964 Helsinki Declaration and its later amendments or comparable ethical standards. The usage of archived diagnostic left-over tissues for TMA manufacturing, the analysis for research purposes, and patient data analysis were approved by the ethics committee, Bonn University Hospital (IRB number 417/17).

Informed Consent Statement: Patient consent was waived due to the retrospective character of the study and the usage of archived diagnostic left-over tissues.

Data Availability Statement: The data presented in this study are available upon reasonable request from the corresponding author.

Acknowledgments: The authors would like to express their gratitude to Kerstin Fuchs and Seher Aktekin for their outstanding technical support.

Conflicts of Interest: The authors declare no conflict of interest.

\section{References}

1. $\quad$ Fitzmaurice, C.; Dicker, D.; Pain, A.; Hamavid, H.; Moradi-Lakeh, M.; MacIntyre, M.F.; Allen, C.; Hansen, G.; Woodbrook, R.; Wolfe, C.; et al. The global burden of cancer 2013. JAMA Oncol. 2015, 1, 505. [CrossRef] [PubMed]

2. Siegel, R.L.; Miller, K.D.; Jemal, A. Cancer statistics, 2020. CA Cancer J. Clin. 2020, 70, 7-30. [CrossRef] [PubMed]

3. Saha, S.K.; Zhu, A.X.; Fuchs, C.S.; Brook, S.G.A. Forty-year trends in cholangiocarcinoma incidence in the U.S.: Intrahepatic disease on the rise. Oncologist 2016, 21. [CrossRef] [PubMed]

4. Valle, J.W.; Borbath, I.; Khan, S.A.; Huguet, F.; Gruenberger, T.; Arnold, D. ESMO guidelines committee biliary cancer: ESMO clinical practice guidelines for diagnosis, treatment and follow-up. Ann. Oncol. Off. J. Eur. Soc. Med. Oncol. 2016, 27, v28-v37. [CrossRef] 
5. $\quad$ Bridgewater, J.; Galle, P.R.; Khan, S.A.; Llovet, J.M.; Park, J.W.; Patel, T.; Pawlik, T.M.; Gores, G.J. Guidelines for the diagnosis and management of intrahepatic cholangiocarcinoma. J. Hepatol. 2014, 60, 1268-1289. [CrossRef]

6. $\quad$ Endo, I.; Gonen, M.; Yopp, A.C.; Dalal, K.M.; Zhou, Q.; Klimstra, D.; D'Angelica, M.; DeMatteo, R.P.; Fong, Y.; Schwartz, L.; et al. Intrahepatic cholangiocarcinoma: Rising frequency, improved survival, and determinants of outcome after resection. Ann. Surg. 2008, 248, 84-96. [CrossRef]

7. Boonstra, K.; Weersma, R.K.; van Erpecum, K.J.; Rauws, E.A.; Spanier, B.W.M.; Poen, A.C.; van Nieuwkerk, K.M.; Drenth, J.P.; Witteman, B.J.; Tuynman, H.A.; et al. Population-based epidemiology, malignancy risk, and outcome of primary sclerosing cholangitis. Hepatology 2013, 58, 2045-2055. [CrossRef]

8. Hasita, H.; Komohara, Y.; Okabe, H.; Masuda, T.; Ohnishi, K.; Lei, X.F.; Beppu, T.; Baba, H.; Takeya, M. Significance of alternatively activated macrophages in patients with intrahepatic cholangiocarcinoma. Cancer Sci. 2010, 101, 1913-1919. [CrossRef]

9. Cadamuro, M.; Morton, S.D.; Strazzabosco, M.; Fabris, L. Unveiling the role of tumor reactive stroma in cholangiocarcinoma: An opportunity for new therapeutic strategies. Transl. Gastrointest. Cancer 2013, 2, 130-144. [CrossRef]

10. McNamara, M.G.; Templeton, A.J.; Maganti, M.; Walter, T.; Horgan, A.M.; McKeever, L.; Min, T.; Amir, E.; Knox, J.J. Neutrophil/lymphocyte ratio as a prognostic factor in biliary tract cancer. Eur. J. Cancer Oxf. Engl. 1990 2014, 50, 1581-1589. [CrossRef]

11. Chen, Q.; Yang, L.-X.; Li, X.-D.; Yin, D.; Shi, S.-M.; Chen, E.-B.; Yu, L.; Zhou, Z.-J.; Zhou, S.-L.; Shi, Y.-H.; et al. The elevated preoperative neutrophil-to-lymphocyte ratio predicts poor prognosis in intrahepatic cholangiocarcinoma patients undergoing hepatectomy. Tumour Biol. J. Int. Soc. Oncodev. Biol. Med. 2015, 36, 5283-5289. [CrossRef]

12. Zhang, Y.; Jiang, C.; Li, J.; Sun, J.; Qu, X. Prognostic significance of preoperative neutrophil/lymphocyte ratio and platelet/lymphocyte ratio in patients with gallbladder carcinoma. Clin. Transl. Oncol. Off. Publ. Fed. Span. Oncol. Soc. Natl. Cancer Inst. Mex. 2015, 17, 810-818. [CrossRef]

13. Lin, G.; Liu, Y.; Li, S.; Mao, Y.; Wang, J.; Shuang, Z.; Chen, J.; Li, S. Elevated neutrophil-to-lymphocyte ratio is an independent poor prognostic factor in patients with intrahepatic cholangiocarcinoma. Oncotarget 2016, 7, 50963-50971. [CrossRef]

14. Yeh, Y.-C.; Lei, H.-J.; Chen, M.-H.; Ho, H.-L.; Chiu, L.-Y.; Li, C.-P.; Wang, Y.-C. C-Reactive Protein (CRP) is a promising diagnostic immunohistochemical marker for intrahepatic cholangiocarcinoma and is associated with better prognosis. Am. J. Surg. Pathol. 2017, 41, 1630-1641. [CrossRef]

15. Rapoport, B.L.; Steel, H.C.; Theron, A.J.; Smit, T.; Anderson, R. Role of the neutrophil in the pathogenesis of advanced cancer and impaired responsiveness to therapy. Molecules 2020, 25, 1618. [CrossRef]

16. Zhang, H.; Liu, H.; Shen, Z.; Lin, C.; Wang, X.; Qin, J.; Qin, X.; Xu, J.; Sun, Y. Tumor-infiltrating neutrophils is prognostic and predictive for postoperative adjuvant chemotherapy benefit in patients with gastric cancer. Ann. Surg. 2018, 267, 311-318. [CrossRef]

17. Zeindler, J.; Angehrn, F.; Droeser, R.; Däster, S.; Piscuoglio, S.; Ng, C.K.Y.; Kilic, E.; Mechera, R.; Meili, S.; Isaak, A.; et al. Infiltration by myeloperoxidase-positive neutrophils is an independent prognostic factor in breast cancer. Breast Cancer Res. Treat. 2019, 177, 581-589. [CrossRef]

18. Brierley, J.D.; Gospodarowicz, M.K.; Wittekind, C. TNM Classification of Malignant Tumours, 8th ed.; John Wiley \& Sons: Hoboken, NJ, USA, 2017; ISBN 978-1-119-26354-8.

19. Zen, Y.; Adsay, N.V.; Bardadin, K.; Colombari, R.; Ferrell, L.; Haga, H.; Hong, S.-M.; Hytiroglou, P.; Klöppel, G.; Lauwers, G.Y.; et al. Biliary intraepithelial neoplasia: An international interobserver agreement study and proposal for diagnostic criteria. Mod. Pathol. 2007, 20, 701-709. [CrossRef]

20. Basturk, O.; Aishima, S.; Esposito, I. Biliary intraepithelial neoplasia. In WHO Classification of Tumours: Digestive System Tumours; WHO Classification of Tumours Editorial Board, International Agency for Research on Cancer: Lyon, France, 2019; Volume 1, ISBN 978-92-832-4499-8.

21. R Core Team. R: A Language and Environment for Statistical Computing; R Core Team: Vienna, Austria, 2020.

22. Kassambara, A.; Kosinski, M.; Biecek, P. Survminer: Drawing Survival Curves Using "Ggplot2", 2020.

23. Masucci, M.T.; Minopoli, M.; Carriero, M.V. Tumor associated neutrophils. Their role in tumorigenesis, metastasis, prognosis and therapy. Front. Oncol. 2019, 9, 1146. [CrossRef]

24. Powell, D.R.; Huttenlocher, A. Neutrophils in the tumor microenvironment. Trends Immunol. 2016, 37, 41-52. [CrossRef]

25. Fridlender, Z.G.; Sun, J.; Kim, S.; Kapoor, V.; Cheng, G.; Ling, L.; Worthen, G.S.; Albelda, S.M. Polarization of tumor-associated neutrophil phenotype by TGF- $\beta$ : “N1" versus “N2" TAN. Cancer Cell 2009, 16, 183-194. [CrossRef]

26. Flavell, R.A.; Sanjabi, S.; Wrzesinski, S.H.; Licona-Limón, P. The polarization of immune cells in the tumour environment by TGF $\beta$. Nat. Rev. Immunol. 2010, 10, 554-567. [CrossRef]

27. Eruslanov, E.B.; Singhal, S.; Albelda, S.M. Mouse versus human neutrophils in cancer: A major knowledge gap. Trends Cancer 2017, 3, 149-160. [CrossRef]

28. Roy, S.; Glaser, S.; Chakraborty, S. Inflammation and progression of cholangiocarcinoma: Role of angiogenic and lymphangiogenic mechanisms. Front. Med. 2019, 6, 293. [CrossRef]

29. Schottenfeld, D.; Beebe-Dimmer, J. Chronic inflammation: A common and important factor in the pathogenesis of Neoplasia. $C A$ Cancer J. Clin. 2006, 56, 69-83. [CrossRef] 
30. Posabella, A.; Köhn, P.; Lalos, A.; Wilhelm, A.; Mechera, R.; Soysal, S.; Muenst, S.; Güth, U.; Stadlmann, S.; Terracciano, L.; et al. High density of CD66B in primary high-grade ovarian cancer independently predicts response to chemotherapy. J. Cancer Res. Clin. Oncol. 2020, 146, 127-136. [CrossRef]

31. Stenzel, P.J.; Schindeldecker, M.; Tagscherer, K.E.; Foersch, S.; Herpel, E.; Hohenfellner, M.; Hatiboglu, G.; Alt, J.; Thomas, C.; Haferkamp, A.; et al. Prognostic and predictive value of tumor-infiltrating leukocytes and of immune checkpoint molecules PD1 and PDL1 in clear cell renal cell carcinoma. Transl. Oncol. 2020, 13, 336-345. [CrossRef]

32. Li, Y.-W.; Qiu, S.-J.; Fan, J.; Zhou, J.; Gao, Q.; Xiao, Y.-S.; Xu, Y.-F. Intratumoral neutrophils: A Poor prognostic factor for hepatocellular carcinoma following resection. J. Hepatol. 2011, 54, 497-505. [CrossRef] [PubMed]

33. Kuang, D.-M.; Zhao, Q.; Wu, Y.; Peng, C.; Wang, J.; Xu, Z.; Yin, X.-Y.; Zheng, L. Peritumoral neutrophils link inflammatory response to disease progression by fostering angiogenesis in hepatocellular carcinoma. J. Hepatol. 2011, 54, 948-955. [CrossRef] [PubMed]

34. Zhou, S.-L.; Dai, Z.; Zhou, Z.-J.; Wang, X.-Y.; Yang, G.-H.; Wang, Z.; Huang, X.-W.; Fan, J.; Zhou, J. Overexpression of CXCL5 mediates neutrophil infiltration and indicates poor prognosis for hepatocellular carcinoma. Hepatology 2012, 56, 2242-2254. [CrossRef] [PubMed]

35. Ino, Y.; Yamazaki-Itoh, R.; Shimada, K.; Iwasaki, M.; Kosuge, T.; Kanai, Y.; Hiraoka, N. Immune cell infiltration as an indicator of the immune microenvironment of pancreatic cancer. Br. J. Cancer 2013, 108, 914-923. [CrossRef]

36. Gu, F.-M.; Gao, Q.; Shi, G.-M.; Zhang, X.; Wang, J.; Jiang, J.-H.; Wang, X.-Y.; Shi, Y.-H.; Ding, Z.-B.; Fan, J.; et al. Intratumoral IL-17+ cells and neutrophils show strong prognostic significance in intrahepatic cholangiocarcinoma. Ann. Surg. Oncol. 2012, 19, 2506-2514. [CrossRef]

37. Mao, Z.-Y.; Zhu, G.-Q.; Xiong, M.; Ren, L.; Bai, L. Prognostic value of neutrophil distribution in cholangiocarcinoma. World J. Gastroenterol. 2015, 21, 4961-4968. [CrossRef]

38. Eruslanov, E.B.; Bhojnagarwala, P.S.; Quatromoni, J.G.; Stephen, T.L.; Ranganathan, A.; Deshpande, C.; Akimova, T.; Vachani, A.; Litzky, L.; Hancock, W.W.; et al. Tumor-associated neutrophils stimulate t cell responses in early-stage human lung cancer. J. Clin. Invest. 2014, 124, 5466-5480. [CrossRef]

39. Wu, P.; Wu, D.; Ni, C.; Ye, J.; Chen, W.; Hu, G.; Wang, Z.; Wang, C.; Zhang, Z.; Xia, W.; et al. Г $\delta T 17$ cells promote the accumulation and expansion of myeloid-derived suppressor cells in human colorectal cancer. Immunity 2014, 40, 785-800. [CrossRef]

40. Singhal, S.; Bhojnagarwala, P.S.; O’Brien, S.; Moon, E.K.; Garfall, A.L.; Rao, A.S.; Quatromoni, J.G.; Stephen, T.L.; Litzky, L.; Deshpande, C.; et al. Origin and role of a subset of tumor-associated neutrophils with antigen-presenting cell features in early-stage human lung cancer. Cancer Cell 2016, 30, 120-135. [CrossRef]

41. Ercolani, G.; Dazzi, A.; Giovinazzo, F.; Ruzzenente, A.; Bassi, C.; Guglielmi, A.; Scarpa, A.; D’Errico, A.; Pinna, A.D. Intrahepatic, peri-hilar and distal cholangiocarcinoma: Three different locations of the same tumor or three different tumors? Eur. J. Surg. Oncol. 2015, 41, 1162-1169. [CrossRef]

42. Cardinale, V.; Renzi, A.; Carpino, G.; Torrice, A.; Bragazzi, M.C.; Giuliante, F.; DeRose, A.M.; Fraveto, A.; Onori, P.; Napoletano, C.; et al. Tumorigenesis and neoplastic progression profiles of cancer stem cell subpopulations in cholangiocarcinomas. Am. J. Pathol. 2015, 185. [CrossRef]

43. Mayr, C.; Ocker, M.; Ritter, M.; Pichler, M.; Neureiter, D.; Kiesslich, T. Biliary tract cancer stem cells-Translational options and challenges. World J. Gastroenterol. 2017, 23, 2470-2482. [CrossRef]

44. Andersen, J.B.; Spee, B.; Blechacz, B.R.; Avital, I.; Komuta, M.; Barbour, A.; Conner, E.A.; Gillen, M.C.; Roskams, T.; Roberts, L.R.; et al. Genomic and Genetic characterization of cholangiocarcinoma identifies therapeutic targets for tyrosine kinase inhibitors. Gastroenterology 2012, 142, 1021-1031.e15. [CrossRef] 